\title{
Dietary Shiitake Mushroom (Lentinus edodes) Prevents Fat Deposition and Lowers Triglyceride in Rats Fed a High-Fat Diet
}

\author{
D. Handayani, J. Chen, B. J. Meyer, and X. F. Huang \\ Metabolic Research Centre, School of Health Sciences and Illawarra Health and Medical Research Institute, University of Wollongong, \\ Wollongong, NSW 2522, Australia
}

Correspondence should be addressed to X. F. Huang, xhuang@uow.edu.au

Received 6 July 2011; Accepted 14 August 2011

Academic Editor: Gianluca Iacobellis

Copyright ( 2011 D. Handayani et al. This is an open access article distributed under the Creative Commons Attribution License, which permits unrestricted use, distribution, and reproduction in any medium, provided the original work is properly cited.

\begin{abstract}
High-fat diet (HFD) induces obesity. This study examined the effects of Shiitake mushroom on the prevention of alterations of plasma lipid profiles, fat deposition, energy efficiency, and body fat index induced by HFD. Rats were given a low, medium, and high $(7,20,60 \mathrm{~g} / \mathrm{kg}=\mathrm{LD}-\mathrm{M}, \mathrm{MD}-\mathrm{M}, \mathrm{HD}-\mathrm{M})$ Shiitake mushroom powder in their high-fat (50\% in kcal) diets for 6 weeks. The results showed that the rats on the HD-M diet had the lowest body weight gain compared to MD-M and LD-M groups $(P<0.05)$. The total fat deposition was significantly lower $(-35 \%, P<0.05)$ in rats fed an HD-M diet than that of HFD group. Interestingly, plasma triacylglycerol (TAG) level was significantly lower $(-55 \%, P<0.05)$ in rats on HD-M than HFD. This study also revealed the existence of negative correlations between the amount of Shiitake mushroom supplementation and body weight gain, plasma TAG, and total fat masses.
\end{abstract}

\section{Introduction}

Obesity is a chronic health problem. It is predicted that by 2015 approximately 2.3 billion adults will become overweight, and more than 700 million will be obese [1]. Obesity causes many complications including dyslipidaemia, diabetes, hypertension, and heart disease. Recently, obesity has also been associated with the increased incidence of many cancers [2]. Dyslipidaemia is a pathological disorder, and current evidence has highlighted not only total cholesterol but also triacylglycerol (TAG) as lipid risk factors in metabolic syndrome.

Recently, community awareness in nutrition as a cornerstone for healthy life style has increased. Functional food is a concept of nutrition, based on the role of reducing the risk of disease. A food will be considered as a functional food if it gives one or more benefits or positive effects to the body beyond adequate nutritional effects in a way that is relevant to either improve the stage of health and well-being and/or reduce the risk of disease [3].

Shiitake mushrooms are the second most popular and the third widely cultivated edible mushroom in the world [4].
Certain components of Shiitake mushroom have hypolipidaemic effect, such as eritadenine and $\beta$-glucan. Although it has been reported that eritadenine extracted from Shiitake mushroom has hypolipidaemic effect, most of these studies emphasize on the mechanism in hypocholesterolaemic effect [5-8] but not the effect on triglyceride. $\beta$-glucan from Shiitake mushroom is a primarily soluble dietary fibre. $\beta$ glucan also present at high levels in graminaceous crops such as barley, rye, and oat and also in bacteria, fungi, and edible mushrooms [9], but the structure can vary from source to source. Studies have reported that oat $\beta$-glucan can increase satiety, reduce food intake, delay nutrition absorption, and reduce plasma lipid levels [10-12]. Although mushroom has been shown to improve lipid profiles [13$15]$, studies in this regard are incomplete. Some studies have reported single-dose effect on obesity-associated metabolic disorders using mushroom powder supplementation [13, 16-18]. However, no data are currently available on the ability of Shiitake mushroom to affect food intake, body fat deposition, and plasma triglyceride concentrations in a dose response manner. 
Studies have shown that high-fat diet-induced obesity in rodents can develop dyslipidaemia, insulin resistance, and altered metabolic regulatory hormones [19-22]. The dietinduced obese animal model mimics human obesity more than other models such as genetic knockout mutants [23]. This study has systematically examined the effects of Shiitake mushroom on plasma lipid profiles, various types of fat depositions, energy efficiency, and body fat index as well as their relationships in a dose-response manner in rats fed a high-fat diet.

\section{Material and Methods}

2.1. Animals and Diet. All experimental procedures were approved by the Animal Ethics Committee of the University of Wollongong, AE 09/01. Forty Wistar rats, at 9 weeks of age, were obtained from animal resource centre (ARC)-Perth, Western Australia, and were given one week acclimatization to their new environment. They were housed two per cage in environmentally controlled conditions (temperature $22^{\circ} \mathrm{C}$, light cycle from 06:00 to 18:00 hours, and dark cycle from 18:00 to 06:00 hours) and had ad libitum access to food and water. All rats were fed standard laboratory nonpurified diet (YS Feeds PTY-Ltd, Young, NSW, Australia) during the acclimatization period. The forty rats were divided into four groups $(n=10)$ and fed $50 \%$ high-fat diets with an addition of nil, low, medium, or high-dose of Shiitake mushroom powder (HFD, $7 \mathrm{~g} / \mathrm{kg} \mathrm{LD-M,} 20 \mathrm{~g} / \mathrm{kg}$ MD-M, or $60 \mathrm{~g} / \mathrm{kg} \mathrm{HD-}$ $\mathrm{M}$, resp.). The dietary intervention was carried out for six weeks. The food was produced from semisynthetic material according to the recommendation of American Institute of Nutrition AIN-93 for rodent [24]. HFD consisted of the following (g/Kg): corn starch, 210 (Goodman Fielder, NSW, Australia); sucrose, 175 (Bella-vista, NSW, Australia); copha, 105 (Peerless food, Victoria, Australia); lard, 105 (Fonterra brand, Victoria, Australia); sunflower oil, 50 (Goodman Fielder, NSW, Australia); gelatine, 50 (Sunny Hills, Qld, Australia); casein, 128 (Fonterra LTD, Auckland, New Zealand); fiber, 51 (Nature, Rosenberg, Germany); mineral, 67 (MP Biomedical, Ohio, USA); vitamin, 13 (MP Biomedical, Ohio, USA). This study used Shiitake mushroom powder containing $30 \% \beta$-glucan $(\mathrm{w} / \mathrm{w})$ analysed with a Megazyme $\beta$-glucan Kit. Compositions of the four diets are shown in Table 1.

2.2. Food Intake, Body Weight, and Body Composition. Animals were weighed weekly throughout the six-week intervention period. Food intake was measured every 24 hours by weighing the amount of total food ( $\mathrm{g}$ ) provided to the rats and subtracting the remaining food $(\mathrm{g})$ in the cage after 24 hours. Following the intervention period, rats were killed via carbon dioxide asphyxiation. The white adipose tissue (WAT) comprised of visceral fat (epididymal, perirenal, and omental fat) and subcutaneous fat (inguinal fat) and brown adipose tissue (BAT) were then dissected out and weighed. The body fat index (BFI) was calculated as the total amount of epididymal, perirenal, omental, and inguinal fat deposits per $100 \mathrm{~g}$ body weight [25]. The energy efficiency ratio (EER)
TABLE 1: Composition of experimental diet.

\begin{tabular}{lcccc}
\hline \multirow{2}{*}{ Component } & \multicolumn{4}{c}{ Group $^{1}$} \\
& HFD & LD-M & MD-M & HD-M \\
\hline Carbohydrate (\%) & 32 & 33 & 33 & 34 \\
Protein (\%) & 16 & 16 & 16 & 16 \\
Fat (\%) & 50 & 49 & 49 & 48 \\
Fibre (\%) & 2 & 2 & 2 & 3 \\
Shiitake mushroom powder & - & 0.7 & 2 & 6 \\
\% (wt: wt) & - & & & \\
Energy density (kJ/g) & 18.8 & 18.8 & 18.8 & 18.4 \\
\hline
\end{tabular}

${ }^{1}$ HFD: high-fat diet; LD-M: low-dose mushroom in HFD; MD-M: medium mushroom in HFD; HD-M: high-dose mushroom in HFD.

was calculated by body weight gain (BWG) per the amount of energy intake $(\mathrm{g} / \mathrm{kJ})[26]$.

2.3. Plasma Lipid Profile. Blood samples were obtained by puncturing the right ventricle of heart after euthanasia and were collected in ethylenediaminetetraacetic-acid- (EDTA-) coated tubes and centrifuged at $3000 \times \mathrm{rpm}$ at $22^{\circ} \mathrm{C}$ for 25 minutes. High-density lipoprotein (HDL) was isolated from plasma with dextran sulphate and magnesium chloride, based on a modified method from Sjoblom and Eklund [27]. Total plasma cholesterol (TC), TAG and HDL cholesterol were measured using the Konelab 20XT with Infinity reagent from Thermo Fisher Scientific (Auburn NSW, Australia).

2.4. Statistical Analysis. Food intake, BWG, EER, BFI, adipose tissue, and plasma lipids were presented as mean and standard errors. Statistical analysis was performed using SPSS software (version 17.0, SPSS Inc, Chicago, Ill, USA). One-way ANOVAs were used, followed by a post hoc TukeyKramer significant differences test for multiple comparisons among the groups. Differences were considered significant when $P<0.05$.

\section{Results}

3.1. Food Intake, Body Weight, and Fat Deposition. No differences in the amount of food or energy intake were found among all groups (Table 2). This study found significant effects of dietary intervention on body weight gain $\left(F_{3,38}=\right.$ $6.204, P=0.002$, Table 2). Rats on a high dose of Shiitake mushroom diet (HD-M group) had $-35 \%$ and $-39 \%$ lower body weight gains than rats on low- and medium-Shiitake mushroom diets, respectively $(P<0.05)$. There was also a trend of lower body weight gain in the HD-M group compared with the HFD group $(-23 \%, P=0.077)$.

Overall, there were significant effects in total fat masses due to dietary interventions $\left(F_{3,38}=4.355, P=0.010\right.$, Table 2). Rats on HD-M diet had significantly lower total fat masses, $-35 \%$ and $-37 \%$, than the HFD and LD-M groups, respectively. Furthermore, HD-M group also had a trend of lower fat accumulation than the MD-M group $(-32 \%, P=$ 0.076 , Figure 1). The white adipose tissue in MD-M group was $-35 \%$ and $-37 \%$ lower than the HFD and LD-M groups, 
TABLE 2: Food intake, energy intake, body weight, and fat deposits of rats fed experimental diets for 6 weeks ${ }^{1}$.

\begin{tabular}{|c|c|c|c|c|c|}
\hline & \multicolumn{4}{|c|}{ Treatment group } & \multirow{2}{*}{ ANOVA } \\
\hline & HFD & LD-M & MD-M & HD-M & \\
\hline Food intake (g/rat/day) & $24 \pm 0.5$ & $24 \pm 0.5$ & $24 \pm 0.6$ & $23 \pm 0.8$ & NS \\
\hline Energy intake ( $\mathrm{kJ} / \mathrm{rat} /$ day $)$ & $455 \pm 9$ & $449 \pm 9$ & $448 \pm 11$ & $423 \pm 14$ & NS \\
\hline Body weight gain & $129 \pm 8^{\mathrm{a}, \mathrm{b}}$ & $141 \pm 9^{b}$ & $150 \pm 14^{\mathrm{b}}$ & $92 \pm 8^{\mathrm{a}}$ & 0.002 \\
\hline \multicolumn{6}{|l|}{ Fat-pad masses ( $g$ ) } \\
\hline Epididymal & $11.2 \pm 1.0^{\mathrm{a}, \mathrm{b}}$ & $12.1 \pm 1.0^{\mathrm{b}}$ & $11.4 \pm 1.2^{\mathrm{a}, \mathrm{b}}$ & $7.9 \pm 0.5^{\mathrm{a}}$ & 0.014 \\
\hline Perirenal & $14.2 \pm 1.1^{\mathrm{a}}$ & $14.3 \pm 1.7^{\mathrm{a}}$ & $12.8 \pm 1.6^{\mathrm{a}, \mathrm{b}}$ & $8.7 \pm 0.8^{\mathrm{b}}$ & 0.015 \\
\hline Omental & $8.4 \pm 0.6^{\mathrm{a}, \mathrm{b}}$ & $10.5 \pm 1.0^{\mathrm{a}}$ & $8.5 \pm 0.7^{\mathrm{a}, \mathrm{b}}$ & $6.7 \pm 0.6^{\mathrm{b}}$ & 0.009 \\
\hline Inguinal fat & $8.6 \pm 1.5^{\mathrm{a}}$ & $6.8 \pm 0.7^{\mathrm{a}, \mathrm{b}}$ & $8.2 \pm 1.7^{\mathrm{a}}$ & $4.2 \pm 0.5^{\mathrm{b}}$ & 0.012 \\
\hline Visceral fat & $33.8 \pm 2.3^{\mathrm{a}, \mathrm{b}}$ & $37.0 \pm 3.7^{\mathrm{b}}$ & $34.0 \pm 3.7^{\mathrm{a}, \mathrm{b}}$ & $23.3 \pm 1.7^{\mathrm{a}}$ & 0.012 \\
\hline WAT & $42.4 \pm 3.4^{\mathrm{a}}$ & $43.8 \pm 4.3^{\mathrm{a}}$ & $40.6 \pm 4.5^{\mathrm{a}, \mathrm{b}}$ & $27.5 \pm 2.1^{\mathrm{b}}$ & 0.011 \\
\hline BAT & $0.58 \pm 0.05$ & $0.52 \pm 0.06$ & $0.54 \pm 0.05$ & $0.44 \pm 0.01$ & NS \\
\hline Total fat masses & $43.0 \pm 3.4^{\mathrm{a}}$ & $44.4 \pm 4.3^{\mathrm{a}}$ & $41.2 \pm 4.5^{\mathrm{a}, \mathrm{b}}$ & $28.0 \pm 2.1^{\mathrm{b}}$ & 0.010 \\
\hline
\end{tabular}

${ }^{1}$ Values are means \pm SEM, $n=10$. Within a row, values with different superscripts are significantly different, $P<0.05$. Abbreviations used: HFD: high-fat diet; LD-M: low-dose mushroom in HFD; MD-M: medium mushroom in HFD; HD-M, high-dose mushroom in HFD.

respectively $\left(F_{3,38}=4.340\right.$, both $\left.P<0.05\right)$. The visceral fat mass (epididymal + perirenal + omental) was the lowest in the HD-M group among all groups $\left(F_{3,38}=4.215, P=\right.$ 0.012 , Table 2). Furthermore, HD-M group had significantly lower perirenal $(-39 \%)$ and inguinal fat $(-52 \%)$ compared to HFD group. Epididymal and omental fat masses were $29 \%$ and $21 \%$ lower in the HD-M group than HFD group $(P=0,05)$. No differences were found in the BAT masses among all groups.

3.2. Body Fat Index (BFI) and Energy Efficiency Ratio (EER). The BFI was significantly different between four diet groups $\left(F_{3,38}=5.86 ; P=0.002\right)$. Results showed that rats on a HD$M$ diet had a significantly lower BFI than the rats on HFD, $\mathrm{LD}-\mathrm{M}$, and MD-M diets $(-35 \%,-33 \%$, and $-30 \%$, resp.). No differences were found in the BFI between the HFD, LD$\mathrm{M}$, and MD-M groups (Figure 2). There was a significant difference in EER among four diet groups $(F=5.425$, $P=0.004$, Figure 3 ). The HD-M group had a significantly lower EER than the LD-M and MD-M groups $(-30 \%$ and $-34 \%$, resp.) but was not significantly different from HFD. Furthermore, there were no statistical differences in EER between the HFD, LD-M, and MD-M groups.

\subsection{Plasma Triacylglycerol, Cholesterol and HDL Concentra-} tions. There was a significant difference in plasma TAG levels between the four groups $\left(F_{3,38}=7.445 ; P=0.001\right)$. The rats on the HD-M diet had significantly lower levels of TAG than HFD, LD-M, and MD-M ( $-55 \%,-41 \%$, and $-46 \%$, resp.). There were no significant differences among the HFD, LD$\mathrm{M}$, and MD-M groups (Figure 4). Plasma total cholesterol levels did not differ between four groups $(P=0.125)$. Although rats on an HD-M diet had the highest level of plasma HDL, no statistical difference was found (HD-M: HFD: LD-M: $\mathrm{MD}-\mathrm{M}=1.26 \pm 0.01: 1.12 \pm 0.02: 0.95 \pm 0.02$ : $0.84 \pm 0.01 \mathrm{mmol} / \mathrm{L})$, respectively.
3.4. Association between the Dosages of Shiitake Mushroom and BWG, WAT, Plasma Lipid, BFI, and EER. This study found that the amount of Shiitake mushroom added in a high-fat diet $(50 \%$ fat in $\mathrm{kcal})$ was negatively associated with the BWG, WAT, TAG, BFI, and EER (Figure 5). No statistical difference was found between the amount of Shiitake mushroom diets and plasma cholesterol and HDL concentrations (data not shown).

\section{Discussion}

This study showed that adding HD-M in a high-energy diet containing $50 \%$ fat can significantly prevent total fat deposition and significantly lower plasma TAG in rats compared with no addition of Shiitake mushroom diet. It was also found that plasma TAG-lowering effect was negatively associated with the amount of Shiitake mushroom supplementations and positively associated with the amount of visceral fat. Studies have been carried out to test plasma TAG-lowering effect by adding mushroom powder into the diets. The results varied, where studies showed a significant effect of lowering plasma TAG [14, 15, 18, 28, 29], whilst other did not $[17,30,31]$. There are a few factors that may explain these differing results. The first is related to the amount of sucrose in the diet which can lead to a high level of plasma TAG in rodents $[17,18]$. High-sucrose diet promotes a hyper-TAG plasma level by increasing hepatic TAG secretion and decreasing TAG removal [32]. A previous study has reported that if the sucrose amount in the diet is greater than $40 \%$ from total energy, the increasing of plasma TAG is difficult to prevent by enriched mushroom powder diet [17]. In this study, the high-fat diet group as controls supplied $15 \%$ sucrose from total energy. Second is the amount of mushroom in the diet. Studies have shown that adding less than $5 \%(\mathrm{wt}: \mathrm{wt})$ mushroom powder in the diet does not significantly decrease plasma TAG, irrespective the sources $[13,14,17,18,30,31,33]$, which is also what 


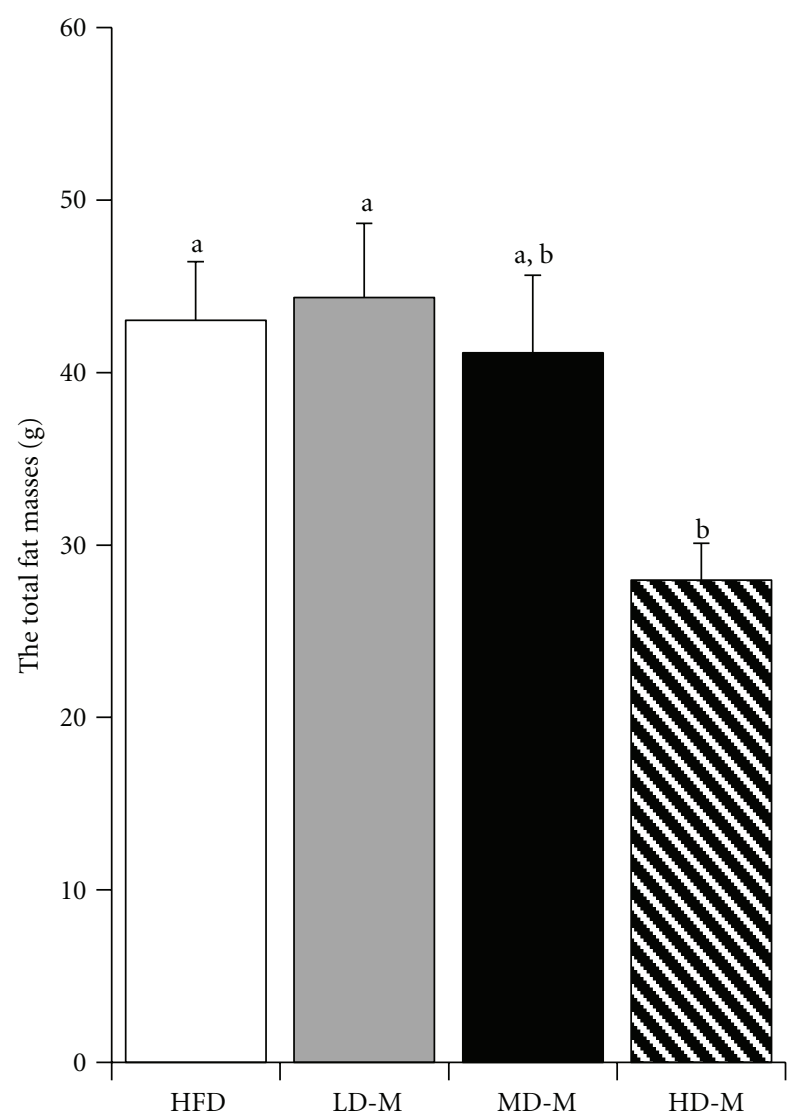

Figure 1: Total fat masses were measured after 6-week dietary treatments in rats. Bars represent means \pm standard errors, $n=10$. ${ }^{\mathrm{ab}}$ Means not sharing a common letter are significantly different among groups at $P<0.05$.

we have found. Therefore, the ratio between the amount of sucrose and enriched mushroom in the diet is important for plasma TAG-lowering effect.

The duration of the dietary intervention using mushroom powder seems less important as it is compared with the dose. Some short-term studies, 3 to 5 weeks $[18,33]$, reported a reduction of plasma TAG concentration, whilst others [16-18] reported no effects. Likewise, the longerintervention studies, such as 6- to 10-week interventions, also showed mixed results with some having no effect [31] and others with positive outcomes to prevent increasing plasma TAG levels $[15,30]$. Hence, the dose of mushroom powder is more important than duration of supplementation.

It is largely unreported to use fat masses as an indicator to determine the effect of adding mushroom to a diet on body weight gain. In animal studies, there are currently limited data relating to the analysis of body fat masses. Kabir and Kimura [30] reported that giving 5\% Shiitake mushroom and $5 \%$ Maitake mushroom for 8 weeks to spontaneous hypertensive rats (HSRs) did not significantly lower adipose tissue compared to control group. There is also a lack of study to define the effect of mushrooms on total fat masses in the human study. In this study, we have demonstrated that the

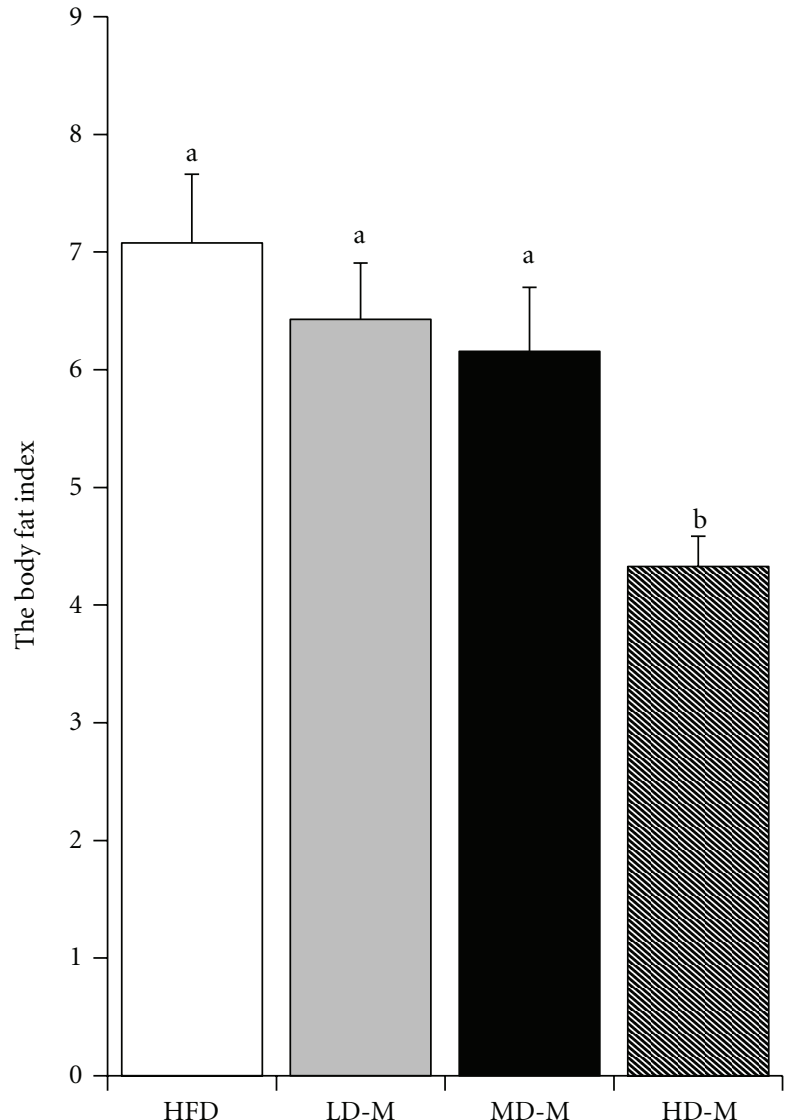

Figure 2: Body fat index was measured after 6-week dietary treatments in rats. Bars represent means \pm standard errors, $n=10$. ${ }^{a b}$ Means not sharing a common letter are significantly different among groups at $P<0.05$.

high-dose Shiitake mushroom lowered total fat deposition. In addition, there was a negative correlation between an increased dosage of Shiitake mushroom and a lower body fat index. Previous study reported that reducing of plasma TAG is associated with the decrease of total fat masses as white adipose tissue [34], which is consistent with our results.

Weight-loss interventions are commonly prescribed for obesity to reduce risk factors associated with metabolic disease. Studies from animal experiments showed various results in terms of adding mushroom powder in diets affecting body weight gain and obesity. For example, a study [14] showed a decrease in body weight gain by $53 \%$ by adding $2 \%$ extracts of Agaricus Blazei mushroom in the diet for 6 weeks in diabetes mellitus Sprague-Dawley rats induced by streptozotocin. Again, body weight gain was significantly reduced by $25 \%$ when adding $20 \%$ Maitake mushroom powder to a hypercholesterolemic diet for 25 weeks [33]. However, no statistical difference in body weight changes was reported in $\mathrm{ApoE}^{-/-}$mice after being given a diet containing $3 \%$ Bunashimeji mushroom powder for $10 \mathrm{wks}$ [31]. A similar result also reported that additional 5\% mushroom powder in the diet for four or eight weeks had no effect on lowering body weight gain in spontaneous hypertension rats 


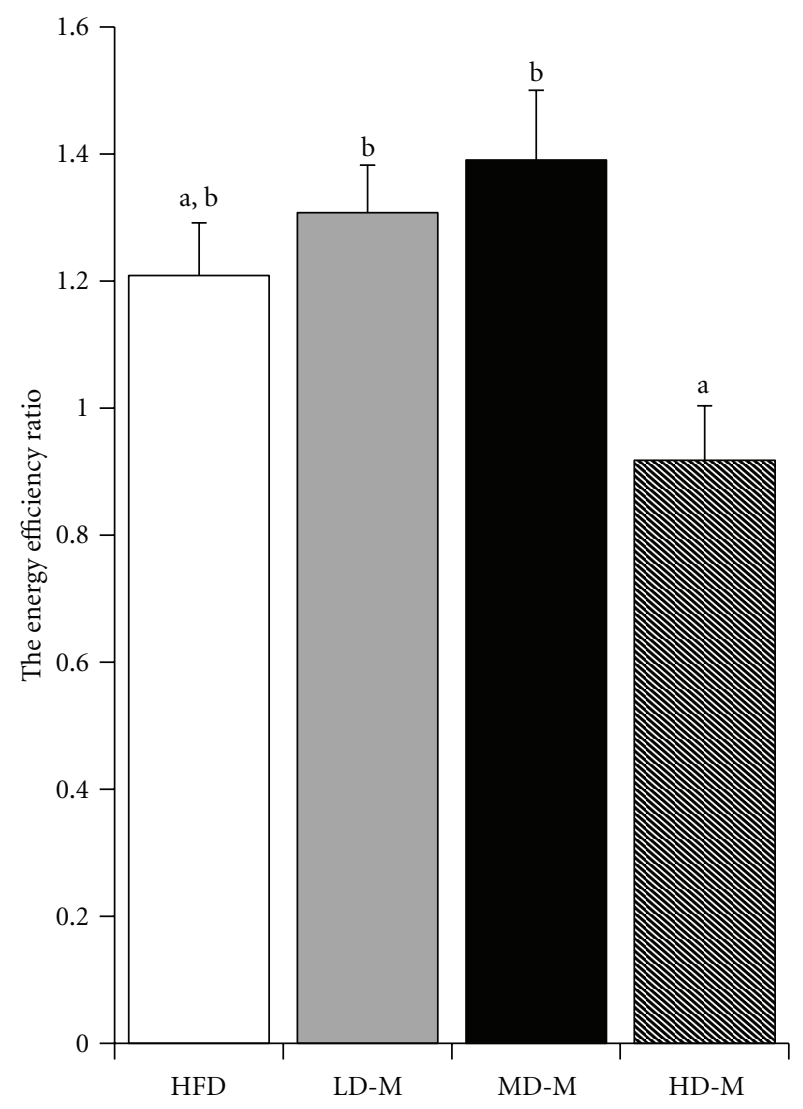

FIGURe 3: Energy efficiency ratio (EER) was measured after 6-week dietary treatments in rats. Bars represent means \pm standard errors, $n=10$. For each variable, labelled means without a common letter differ, $P<0.05$.

or male F344/DuCrj rats $[17,30]$. It appears that different species of animal respond differently in body weight gain to the dietary intervention using mushroom powder. The dosages less than $5 \%$ mushroom powder seem to be less likely to give any favourable effect to prevent body weight gain. The initial body weight and age of animal model seem to have no affect on the outcome of mushroom treatment to prevent the increase of body weight $[16-18,30]$.

This study showed that body weight gain was significantly lower in the HD-M group than the MD-M and LD$\mathrm{M}$ groups. This suggests that there was a threshold effect in terms of the amount of Shiitake mushroom intake for the prevention of weight gain in rats on a high-fat diet. As an estimate of HD-M diet in rats, $90 \mathrm{~g}$ mushroom powder per day may be required for humans to prevent body weight gain and lower TAG. However, this must be tested in humans before the recommendation is given. On the other hand, a low-dose mushroom diet may not be strong enough to prevent weight gain. For example, studies have shown that supplementation of the diet with $30 \mathrm{~g}$ Maitake mushroom per day for 21 days did not achieve body weight lowering effect in patients with moderate dyslipidaemia [35].

This study found a diet of HD-M significantly lowered the energy efficiency ratio (EER) compared to LD-M and

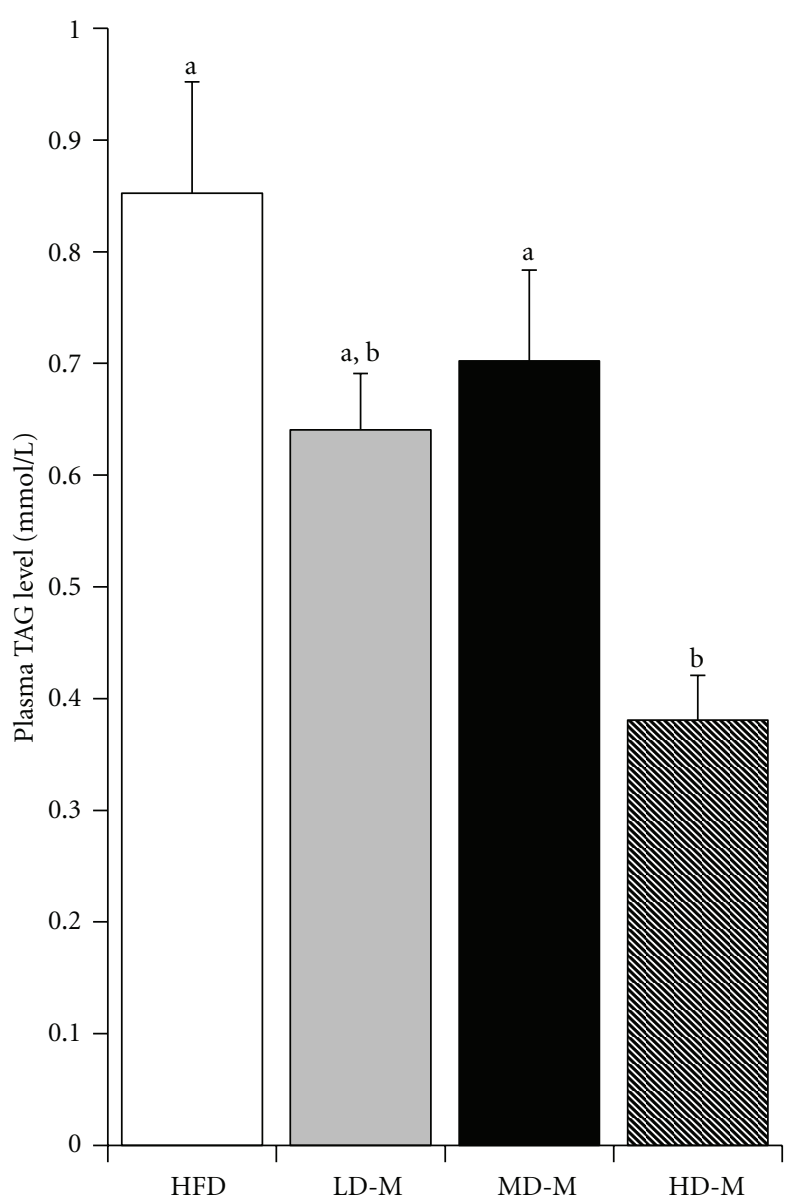

Figure 4: Plasma TAG level was measured after 6-week dietary treatments in rats. Bars represent means \pm standard errors, $n=10$. For each variable, labelled means without a common letter differ, $P<0.05$.

MD-M diets. The HD-M also tended to lower EER when compared with HFD. This is in agreement with a previous study showing that high-dose Shiitake mushroom diet lowers energy efficiency in rats on a high-fat diet as adding Maitake mushroom (20\%) into hypercholesterolemic diet for 4 weeks reduces $27 \%$ energy efficiency in body weight gain in rats [33]. Unfortunately, the study of this kind has not been carried out in humans.

The exact mechanism is not clear how Shiitake mushroom diet resulting in lowering body weight gain, fat masses and plasma TAG. There are three possible mechanisms worthwhile of considerations. Firstly, Shiitake mushroom could increase the fat elimination into faeces. Shiitake mushroom contains $30 \% \beta$-glucan. As a soluble fibre, $\beta$ glucan may enhance luminal viscosity, delay gastric emptying, and/or reduce intestinal absorption. Hence, it decreases fat absorption. Secondly, Shiitake mushroom could reduce nonesterified fatty acid (NEFA) derived from TAG as a source of adipose tissue. Lipolysis of chylomicron and VLDL-TAG are major determinants of adipose tissue development, and the reduced WAT may be affected by the low-NEFA source that enters to adipose tissue. A low NEFA in adipose tissue is 


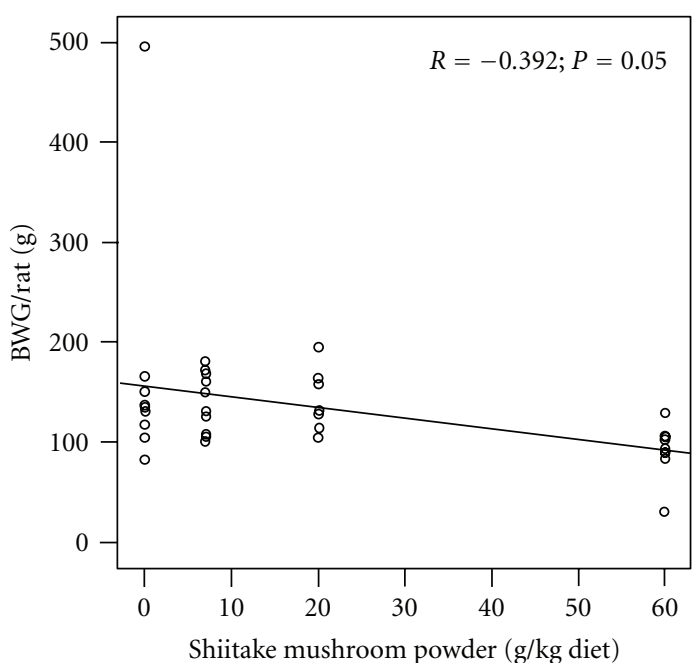

(a)

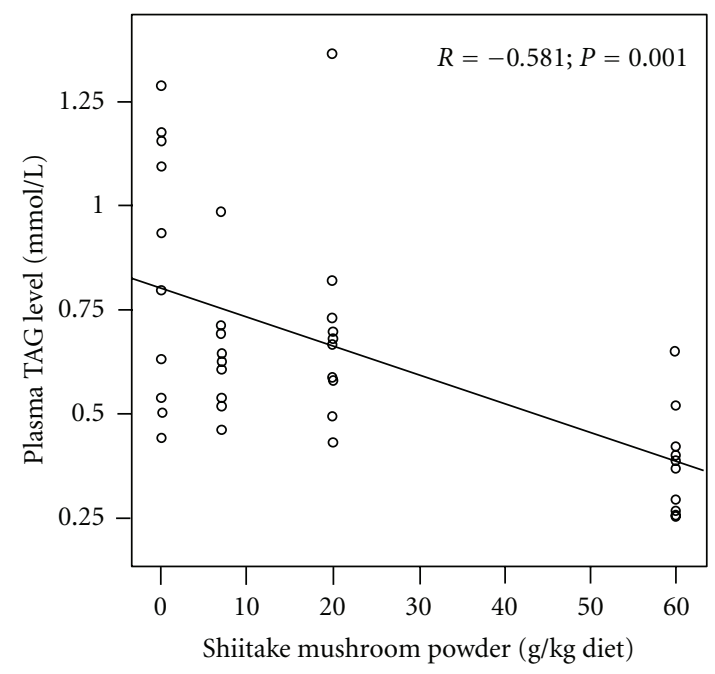

(c)

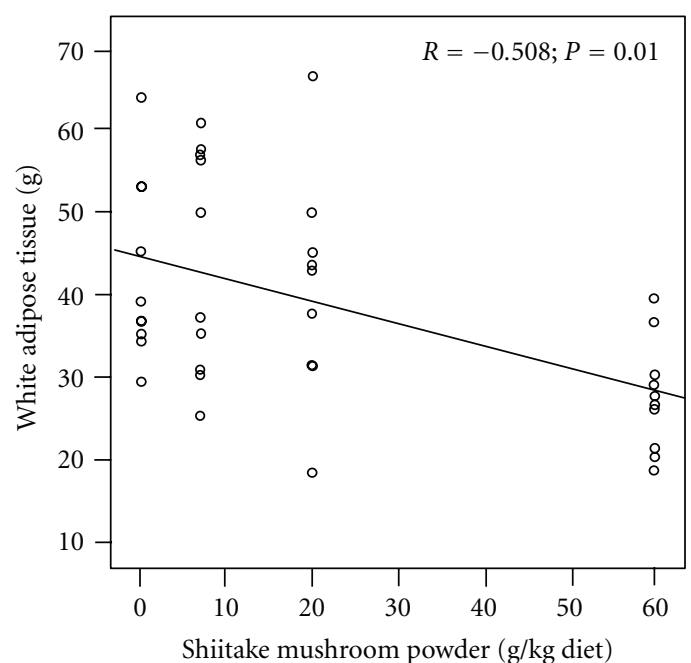

(b)

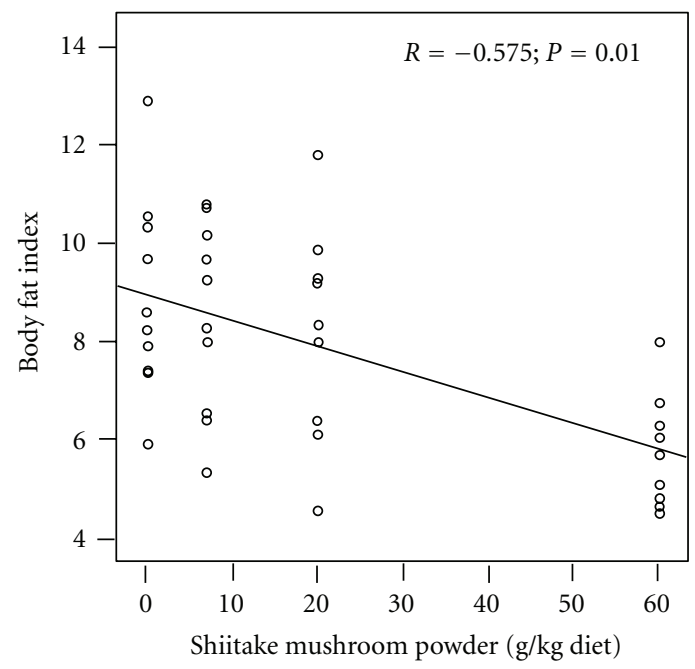

(d)

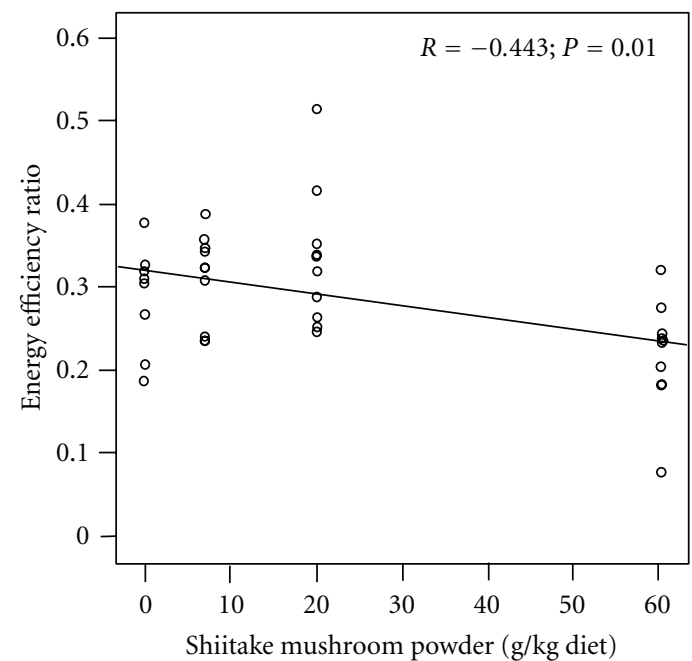

(e)

FIGURE 5: Significant correlations were found between dosages and BWG (a), WAT (b), plasma TAG level (c), BFI (d), and (e) EER. BWG: body weight gain; WAT: white adipose tissue; TAG: triacylglycerol; BFI: body fat index; EER: energy efficiency ratio. 
possibly affected by the activity of enzyme LPL as main gate keeper entry into adipose tissue [34]. The overexpression of LPL in muscle prevents HFD-induced lipid accumulation in adipose tissue. Increased LPL in skeletal muscle leads to an enhanced TAG storage in muscle and then diverts lipoprotein TAG-derived NEFA away from storage in adipose tissue to oxidation in the muscle [36]. Normally fat from food is released into the circulation as lipoprotein-TAG [34]. Thirdly, the possibility that eritadenine from Shiitake mushroom could inhibit the release of triacylglycerol from the liver. The eritadenine has been reported to inhibit the production of phosphatidylcholine that influences the release of VLDL and HDL from liver [5]. Studies showed that adding eritadenine extracted from Shiitake mushroom in the diet can decrease plasma TAG by $27 \%$ in a cholesterol diet, $20 \%$ in cholesterol free diet, and $2 \%$ in diet with choline chloride added [5-7]. The $50 \mathrm{mg}$ eritadenine per $\mathrm{kg}$ diet was shown to be sufficient to elicit hypolipidaemic effect in rats [8]. The concentration of eritadenine is about $0.36 \%$ in Shiitake mushroom powder [37]. The HD-M diet used in this study contains $\sim 200 \mathrm{mg} / \mathrm{kg}$ food. Since TAG was low in HD-M diet, it will be interesting to examine TAG in various organs to ensure its possible storage or exclusion. In view of that, fat content in faecal, plasma NEFA concentration, and accumulation of TAG in the liver and muscle will need to be examined to verify its potential side effects.

In conclusion, adding high Shiitake mushroom in a high-fat diet can prevent body weight gain, fat deposition, and plasma TAG in rats, while further study is necessary to elucidate the underlying mechanisms and the possibility component of Shiitake mushroom of these important effects. Thereby, it could be used as a functional food to prevent obesity. This study encourages an effort pursuing human clinical trial using Shiitake mushroom for prevention and treatment of obesity and its related metabolic disorders.

\section{Abbreviations}

HFD: High fat diet

LD-M: low dose mushroom

MD-M: Medium dose mushroom

HD-M: High dose mushroom

TAG: Triacylglycerol

ARC: Animal resource centre

WAT: White adipose tissue

BAT: Brown adipose tissue

BFI: Body fat index

EER: Energy efficiency ratio

EDTA: Ethylenediaminetetraacetic acid

HDL: High density lipoprotein

TC: $\quad$ Total cholesterol

ANOVA: Analysis of variance

\section{Author Discosure}

D. Handayani, J. Chen, BJ Meyer and XF. Huang have no conflict of interest.

\section{Acknowledgments}

The technical assistance of Mrs. Yizhen Wu is gratefully acknowledged. The project is funded by the Research Council of the University of Wollongong.

\section{References}

[1] WHO, "Media centre obesity and overweight," WHO, 2006.

[2] X. F. Huang and J. Z. Chen, "Obesity, the PI3K/Akt signal pathway and colon cancer," Obesity Reviews, vol. 10, no. 6, pp. 610-616, 2009.

[3] M. B. Roberfroid, "A European consensus of scientific concepts of functional foods," Nutrition, vol. 16, no. 7-8, pp. 689$691,2000$.

[4] S. T. Chang, "The world mushroom industry: trends and technological development," International Journal of Medicinal Mushrooms, vol. 8, no. 4, pp. 297-314, 2006.

[5] Y. Shimada, T. Morita, and K. Sugiyama, "Eritadenineinduced alterations of plasma lipoprotein lipid concentrations and phosphatidylcholine molecular species profile in rats fed cholesterol-free and cholesterol-enriched diets," Bioscience, Biotechnology and Biochemistry, vol. 67, no. 5, pp. 996-1006, 2003.

[6] K. Sugiyama, A. Yamakawa, H. Kawagishi, and S. Saeki, "Dietary eritadenine modifies plasma phosphatidylcholine molecular species profile in rats fed different types of fat," Journal of Nutrition, vol. 127, no. 4, pp. 593-599, 1997.

[7] K. Takashima, C. Sato, and Y. Sasaki, "Effect of eritadenine on cholesterol metabolism in the rat," Biochemical Pharmacology, vol. 23, no. 2, pp. 433-438, 1974.

[8] K. Sugiyama, T. Akachi, and A. Yamakawa, "Eritadenineinduced alteration of hepatic phospholipid metabolism in relation to its hypochlelsterolemic action in rats," Journal of Nutritional Biochemistry, vol. 6, no. 2, pp. 80-87, 1995.

[9] J. Chen and R. Seviour, "Medicinal importance of fungal $\beta$ $(1 \rightarrow 3)$, (1 $\rightarrow 6)$-glucans," Mycological Research, vol. 111, no. 6, pp. 635-652, 2007.

[10] A. L. Jenkins, D. J. A. Jenkins, U. Zdravkovic, P. Würsch, and V. Vuksan, "Depression of the glycemic index by high levels of $\beta$ glucan fiber in two functional foods tested in type 2 diabetes," European Journal of Clinical Nutrition, vol. 56, no. 7, pp. 622628, 2002.

[11] K. R. Juvonen, A. K. Purhonen, M. Salmenkallio-Marttila et al., "Viscosity of oat bran-enriched beverages influences gastrointestinal hormonal responses in healthy humans," Journal of Nutrition, vol. 139, no. 3, pp. 461-466, 2009.

[12] J. Chen and X. F. Huang, "The effects of diets enriched in beta-glucans on blood lipoprotein concentrations," Journal of Clinical Lipidology, vol. 3, no. 3, pp. 154-158, 2009.

[13] P. C. K. Cheung, "Plasma and hepatic cholesterol levels and fecal neutral sterol excretion are altered in hamsters fed straw mushroom diets," Journal of Nutrition, vol. 128, no. 9, pp. 1512-1516, 1998.

[14] Y. W. Kim, K. H. Kim, H. J. Choi, and D. S. Lee, "Anti-diabetic activity of $\beta$-glucans and their enzymatically hydrolyzed oligosaccharides from Agaricus blazei," Biotechnology Letters, vol. 27, no. 7, pp. 483-487, 2005.

[15] C. Xu, Z. HaiYan, Z. JianHong, and G. Jing, "The pharmacological effect of polysaccharides from Lentinus edodes on the oxidative status and expression of VCAM-1mRNA of thoracic aorta endothelial cell in high-fat-diet rats," Carbohydrate Polymers, vol. 74, no. 3, pp. 445-450, 2008. 
[16] M. Fukushima, M. Nakano, Y. Morii, T. Ohashi, Y. Fujiwara, and K. Sonoyama, "Hepatic LDL receptor mRNA in rats is increased by dietary mushroom (Agaricus bisporus) fiber and sugar beet fiber," Journal of Nutrition, vol. 130, no. 9, pp. 21512156, 2000.

[17] M. Fukushima, T. Ohashi, Y. Fujiwara, K. Sonoyama, and M. Nakano, "Cholesterol-lowering effects of maitake (Grifola frondosa) fiber, shiitake (Lentinus edodes) fiber, and enokitake (Flammulina velutipes) fiber in rats," Experimental Biology and Medicine, vol. 226, no. 8, pp. 758-765, 2001.

[18] S. C. Jeong, Y. T. Jeong, B. K. Yang et al., "White button mushroom (Agaricus bisporus) lowers blood glucose and cholesterol levels in diabetic and hypercholesterolemic rats," Nutrition Research, vol. 30, no. 1, pp. 49-56, 2010.

[19] R. Buettner, K. G. Parhofer, M. Woenckhaus et al., "Defining high-fat-diet rat models: metabolic and molecular effects of different fat types," Journal of Molecular Endocrinology, vol. 36, no. 3, pp. 485-501, 2006.

[20] R. Buettner, J. Schölmerich, and L. C. Bollheimer, "High-fat diets: modeling the metabolic disorders of human obesity in rodents," Obesity, vol. 15, no. 4, pp. 798-808, 2007.

[21] S. Lin, T. C. Thomas, L. H. Storlien, and X. F. Huang, "Development of high fat diet-induced obesity and leptin resistance in C57B1/6J mice," International Journal of Obesity, vol. 24, no. 5, pp. 639-646, 2000.

[22] D. A. Ainslie, J. Proietto, B. C. Fam, and A. W. Thorburn, "Short-term, high-fat diets lower circulating leptin concentrations in rats," American Journal of Clinical Nutrition, vol. 71, no. 2, pp. 438-442, 2000.

[23] M. Van Heek, D. S. Compton, C. F. France et al., "Diet-induced obese mice develop peripheral, but not central, resistance to leptin," Journal of Clinical Investigation, vol. 99, no. 3, pp. 385390, 1997.

[24] P. G. Reeves, "Components of the AIN-93 diets as improvements in the AIN-76A diet," Journal of Nutrition, vol. 127, no. 5, pp. 838S-841S, 1997.

[25] H. Wang, L. H. Storlien, and X. -F. Huang, "Effects of dietary fat types on body fatness, leptin, and ARC leptin receptor, NPY, and AgRP mRNA expression," American Journal of Physiology, vol. 282, pp. E1352-E1359, 2002.

[26] D. Shin, "The effect of seamustard on blood lipid profiles and glucose level of rats fed diet with different energy composition," Nutrition Research and Practice, vol. 3, pp. 3137, 2009.

[27] L. Sjoblom and A. Eklund, "Determination of HDL2 cholesterol by precipitation with dextran sulfate and magnesium chloride: establishing optimal conditions for rat plasma," Lipids, vol. 24, no. 6, pp. 532-534, 1989.

[28] M. Kabir, J. M. Oppert, H. Vidal et al., "Four-week lowglycemic index breakfast with a modest amount of soluble fibers in type 2 diabetic men," Metabolism: Clinical and Experimental, vol. 51, no. 7, pp. 819-826, 2002.

[29] N. A. Talpur, B. W. Echard, A. Y. Fan, O. Jaffari, D. Bagchi, and H. G. Preuss, "Antihypertensive and metabolic effects of whole Maitake mushroom powder and its fractions in two rat strains," Molecular and Cellular Biochemistry, vol. 237, no. 1-2, pp. 129-136, 2002.

[30] Y. Kabir and S. Kimura, "Dietary mushrooms reduce blood pressure in spontaneously hypertensive rats (SHR)," Journal of Nutritional Science and Vitaminology, vol. 35, no. 1, pp. 91-94, 1989.

[31] K. Mori, C. Kobayashi, T. Tomita, S. Inatomi, and M. Ikeda, "Antiatherosclerotic effect of the edible mushrooms
Pleurotus eryngii (Eringi), Grifola frondosa (Maitake), and Hypsizygus marmoreus (Bunashimeji) in apolipoprotein Edeficient mice," Nutrition Research, vol. 28, no. 5, pp. 335-342, 2008.

[32] C. Y. Xue, H. Kageyama, M. Kashiba et al., "Different origin of hypertriglyceridemia induced by a high-fat and a high-sucrose diet in ventromedial hypothalamic-lesioned obese and normal rats," International Journal of Obesity, vol. 25, no. 3, pp. 434438, 2001.

[33] K. Kubo and H. Nanba, "The effect of maitake mushrooms on liver and serum lipids," Alternative Therapies in Health and Medicine, vol. 2, no. 5, pp. 62-66, 1996.

[34] P. J. Voshol, P. C. N. Rensen, K. W. van Dijk, J. A. Romijn, and L. M. Havekes, "Effect of plasma triglyceride metabolism on lipid storage in adipose tissue: studies using genetically engineered mouse models," Biochimica et Biophysica Acta, vol. 1791, no. 6, pp. 479-485, 2009.

[35] I. Schneider, G. Kressel, A. Meyer, U. Krings, R. G. Berger, and A. Hahn, "Lipid lowering effects of oyster mushroom (Pleurotus ostreatus) in humans," Journal of Functional Foods, vol. 3, no. 1, pp. 17-24, 2011.

[36] D. R. Jensen, I. R. Schlaepfer, C. L. Morin et al., "Prevention of diet-induced obesity in transgenic mice overexpressing skeletal muscle lipoprotein lipase," American Journal of Physiology, vol. 273, pp. R683-R689, 1997.

[37] J. Enman, U. Rova, and K. A. Berglund, "Quantification of the bioactive compound eritadenine in selected strains of shiitake mushroom (Lentinus edodes)," Journal of Agricultural and Food Chemistry, vol. 55, no. 4, pp. 1177-1180, 2007. 


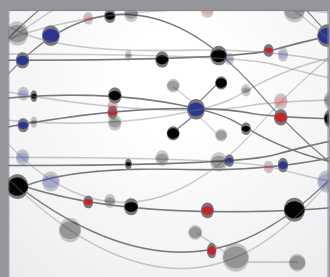

The Scientific World Journal
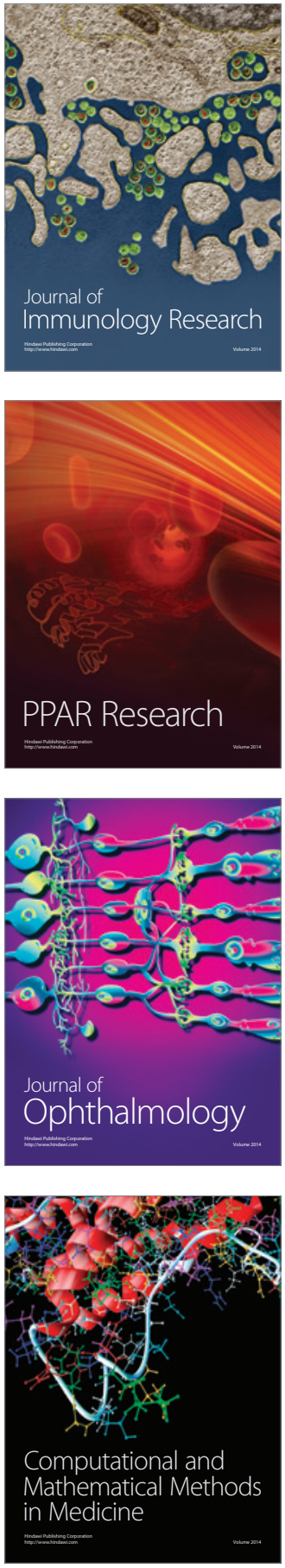

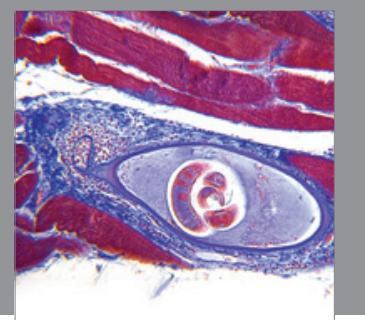

Gastroenterology

Research and Practice
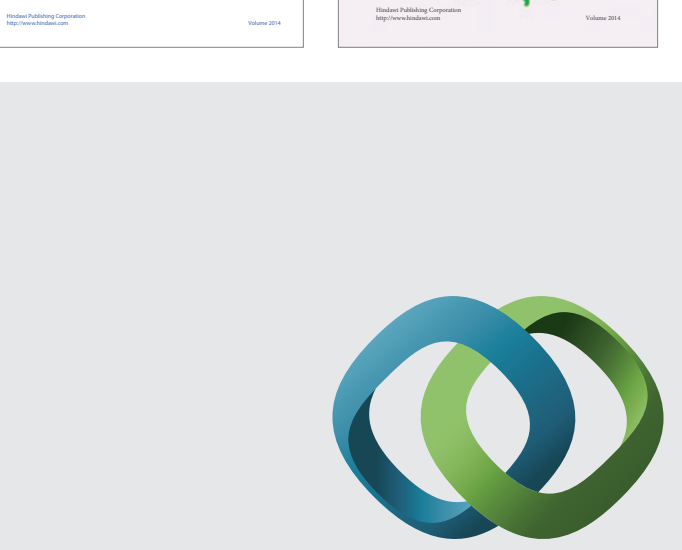

\section{Hindawi}

Submit your manuscripts at

http://www.hindawi.com
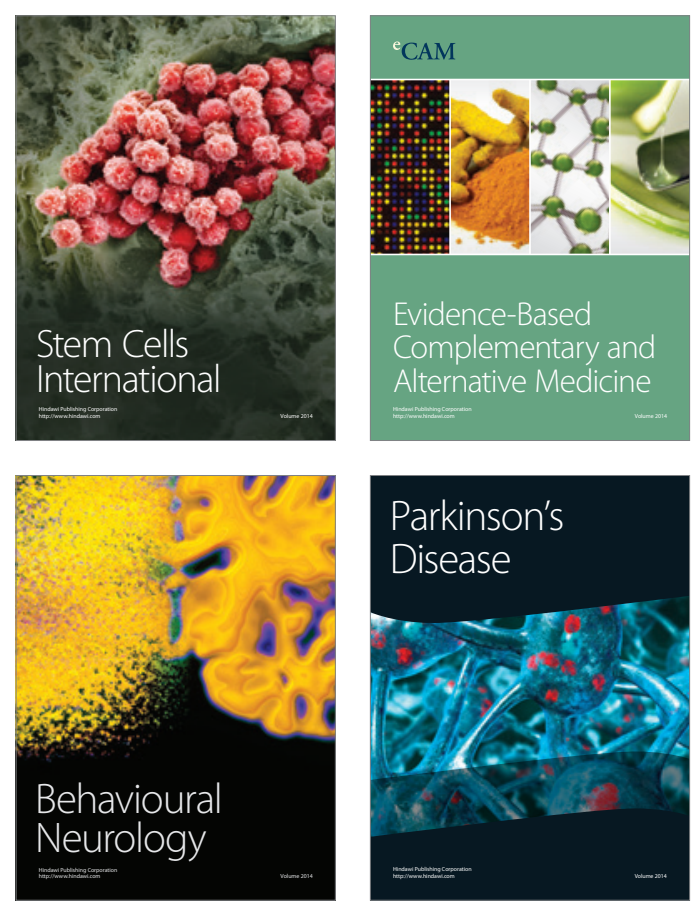

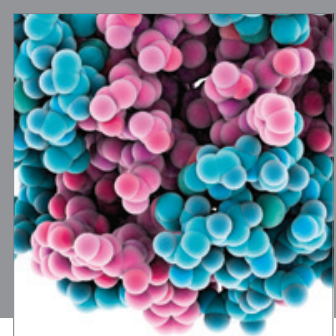

Journal of
Diabetes Research

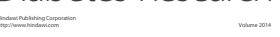

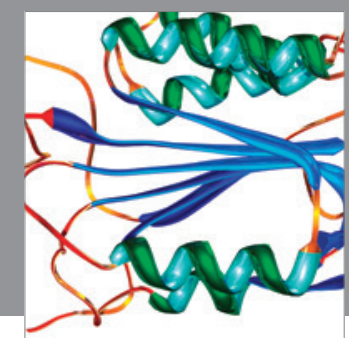

Disease Markers
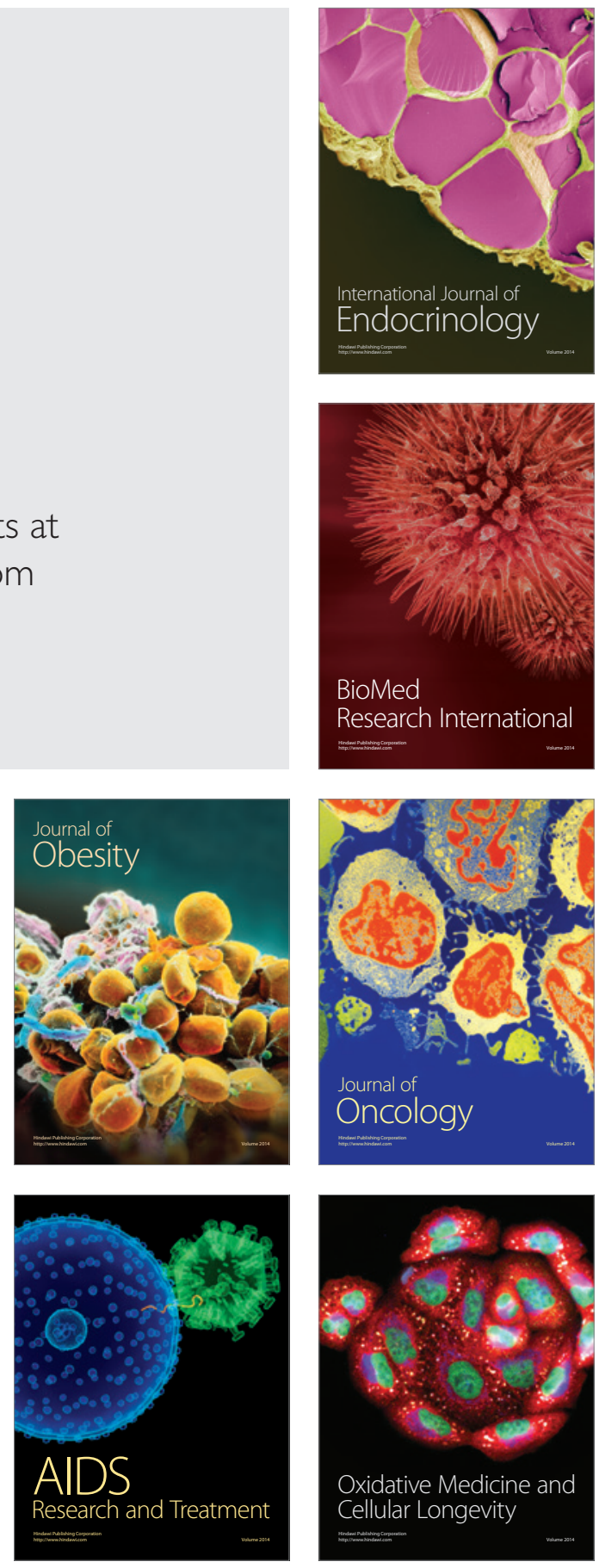